\title{
COMMUNICATION
}

Cite this: DOI:

$10.1039 / \times 0 \times x 00000 x$

Received 00 th January 2012 ,

Accepted 00 th January 2012

DOI : $10.1039 / \times 0 \times x 00000 x$

www.rsc.org/

\section{Observations on transition metal free biaryl coupling: Potassium tert-butoxide alone promotes the reaction without diamine or phenanthroline catalysts}

\author{
J. Cuthbertson, ${ }^{a}$ V. J. Gray ${ }^{a}$ and Jonathan D. Wilden* ${ }^{a}$
}

Biaryl coupling (often labelled 'C-H activation') of aromatic systems can be achieved by potassium tert-butoxide alone in the absence of any amine or bipyridine catalyst $(1,10-$ phenanthroline or $N, N$ '-dimethylethylenediamine being the most common), previously reported to be essential. Various mechanistic studies and observations are presented which suggest that when 1,10-phenanthroline is employed as the catalyst, the alkoxide is destroyed almost immediately.

One of the most important observations in organic chemistry in recent years has been the discovery that certain reactions previously thought the preserve of transition metal catalysis (for example C-H activation, ${ }^{1}$ biaryl couplings, ${ }^{2,3}$ certain $\mathrm{Heck}^{4}$ and Sonogashira ${ }^{5}$ processes) can be effected without the requirement for a transition metal. These quite remarkable observations have raised many questions in the academic community as to the origin of these reactions. In some cases trace metal contaminants in coreagents have been shown to be the origin of the catalysis ${ }^{6}$ while in others a radical mechanism has been implicated. ${ }^{2}$ The common feature of those reactions where a TM is not required is the requirement of either a phenanthroline or diamine derivative to effect the catalysis (with phenanthroline derivatives being used most frequently)., 3 The pioneering work of Hayashi ${ }^{2}$ and, Kwong and Lei $^{3}$ which demonstrates this is outlined in Scheme 1. Since their publications in 2010, numerous similar approaches have followed.
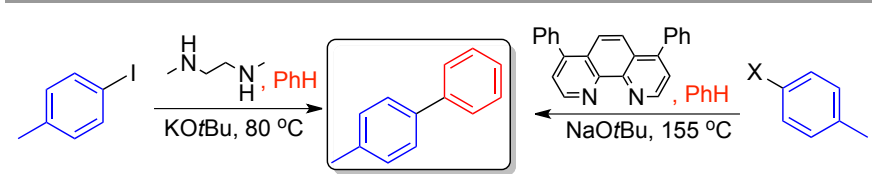

Scheme 1. TM free biaryl coupling with amine catalysis

We here report observations from our own laboratory, which demonstrate that the amine or phenanthroline additive is not an essential component of these $\mathrm{C}-\mathrm{H}$ activation and biaryl coupling reactions. In some cases the additive may improve the rate or efficiency of the reaction but the reactions still proceed in their absence. This paper outlines our experiments, observations and thoughts as to why this is the case.

\begin{tabular}{|c|c|c|c|c|c|}
\hline \multirow[b]{2}{*}{ Entry } & \multicolumn{2}{|c|}{$3 \mathrm{~h}$, additive, $\Delta$} & \multirow[b]{2}{*}{ Additive } & \multicolumn{2}{|c|}{$\begin{array}{l}\text { 1,10-Phenanthroline } \\
\text { (Phen) } \\
N, N^{\prime} \text {-Dimethylethylene- } \\
\text { diamine (DMEDA) }\end{array}$} \\
\hline & $\mathrm{X}$ & $M$ & & Temp ${ }^{\circ} \mathrm{C}$ & Yield $\%^{a}$ \\
\hline 1 & $\mathrm{I}$ & $\mathrm{K}$ & - & 55 & 0 \\
\hline 2 & I & K & - & 85 & 0 \\
\hline 3 & I & $\mathrm{K}$ & - & 110 & 3 \\
\hline 4 & I & $\mathrm{K}$ & - & 160 & 38 \\
\hline 5 & I & K & - & 160 & $66^{\mathrm{b}}$ \\
\hline 6 & $\mathrm{Br}$ & $\mathrm{K}$ & - & 160 & 6 \\
\hline 7 & $\mathrm{Cl}$ & $\mathrm{K}$ & - & 160 & 0 \\
\hline 8 & I & $\mathrm{Na}$ & - & 160 & 2 \\
\hline 9 & I & $\mathrm{Li}$ & - & 160 & 0 \\
\hline 10 & I & $\mathrm{Na}$ & 1,10-Phen & 160 & 65 \\
\hline 11 & I & $\mathrm{Na}$ & 15-Crown-5 & 160 & 2 \\
\hline 12 & 1 & K & 1,10-Phen & 85 & 2 \\
\hline 13 & I & K & 1,10-Phen & 160 & 65 \\
\hline 14 & I & $\mathrm{K}$ & DMEDA & 85 & $53^{b}$ \\
\hline 15 & I & $\mathrm{K}$ & DMEDA & 160 & 67 \\
\hline
\end{tabular}

${ }^{\mathrm{a}}$ Isolated yields. ${ }^{\mathrm{b}}$ Reaction time extended to $6 \mathrm{~h}$

Our laboratory has long been interested in the reactions of alkoxy bases and their application in TM-free processes. Our previous work has suggested that potassium tert-butoxide undergoes sp-displacement reactions due to the fact that the $\mathrm{O}-\mathrm{K}$ bond is relatively weak and supplies an essentially dissociated alkoxide anion. ${ }^{8}$ We therefore examined the reaction of simple aryl iodides 
with benzene under various conditions. The results are given in Table 1.

The results demonstrate that while amine additives may allow the reactions to proceed under slightly milder conditions, the major effect can be observed without an amine 'ligand' or a transition metal present in the reaction mixture. The experiments also demonstrate the superiority of the potassium cation for effecting these reactions. As has been previously noted, addition of radical traps such as $\mathrm{TEMPO}^{2,3}$ or even prolonged exposure to molecular oxygen attenuates the reaction and so a radical process (the mechanism of which will be discussed later) can be inferred.

Having identified suitable reaction conditions for the process we then turned to examine the scope of the reaction with a variety of substrates. The results are presented in Table 2 .

Table 2: Substrate Scope in TM and amine free $\mathrm{KO} t \mathrm{Bu}$ mediated $\mathrm{C}-\mathrm{H}$ activation.

Entry

We were pleased to observe that the reaction could be effected with a variety of electron rich and electron deficient aryl iodides and that minimal side products were observed. We were also interested to note that addition of the diamine had no effect on the reaction yield but (as shown in Table 1) does allow the reaction to proceed at a much lower temperature.
Along with others working in this field we have been intrigued by the reaction mechanism of these processes. Previous postulated mechanisms have suggested that phenanthroline acts as a bidentate ligand for the alkali metal leading to a chelate that is capable of undergoing electron transfer to an organic substrate to initiate a radical coupling reaction with benzene. ${ }^{2}$

Given that 1,10-phenanthroline is only a weak ligand for the lower Group 1 metal ions ${ }^{9}$ (due to their large ionic radius) an alternative explanation seems likely given that the results Table 1 demonstrates that there is little advantage to employing phenanthroline with the potassium counterion but a significant advantage when sodium tert-butoxide is employed (entries 5, 8, 10 and 13). We therefore decided to examine the role of phenanthroline in these reactions more closely.

We were at first suspicious that the quality of commercially sourced 1,10-phenanthroline was poor. A number of different suppliers supplied the reagent with a faint pink hue, which we believed may be transition metal contaminants. Additionally the reagent contained at least one mole of water, which could be clearly observed in the ${ }^{1} \mathrm{H}$ NMR spectrum. This observation was reflected in the melting point of the material which at $90-99{ }^{\circ} \mathrm{C}$ was significantly below the literature value of $117-118{ }^{\circ} \mathrm{C}$ and almost perfectly matched the literature value for the hydrated species. The elemental analysis data also suggested the hydrate. In order to rectify this, the reagent was converted to the mesylate salt, then washed with concentrated aqueous ammonia before recrystalisation to give the pure material as a white crystalline solid. This material was spectroscopically pure, and gave satisfactory elemental analysis data along with a melting point of $115-116{ }^{\circ} \mathrm{C}$, in good agreement with the literature value for the pure material (Table 3 ).

Table 3: Physical data for unpurified and purified 1,10-phenanthroline

\begin{tabular}{cccc}
\hline Element & $\begin{array}{c}\text { Theoretical } \\
(\%)\end{array}$ & $\begin{array}{c}\text { Unpurified } \\
\left(\mathrm{m} . \mathrm{p} .90-99^{\circ} \mathrm{C}\right)\end{array}$ & $\begin{array}{c}\text { Purified } \\
\text { (m.p. 115-116 } \mathrm{C})\end{array}$ \\
\hline $\mathrm{C}$ & 79.78 & 72.62 & 78.09 \\
$\mathrm{H}$ & 4.47 & 4.89 & 4.57 \\
$\mathrm{~N}$ & 15.55 & 14.12 & 16.2 \\
\hline
\end{tabular}

The first striking observation in these reactions was the immediate, exothermic and vigorous reaction between equimolar mixtures of $\mathrm{KO} t \mathrm{Bu}$ and purified 1,10phen in THF, forming a black tar. Performing the same reaction with unpurified 1,10phenanthroline led to a comparatively more sluggish reaction and a pale yellow solution after five minutes (Figure 1). It is also noteworthy that this reaction occurs even when the aryl iodide is omitted from the mixture.
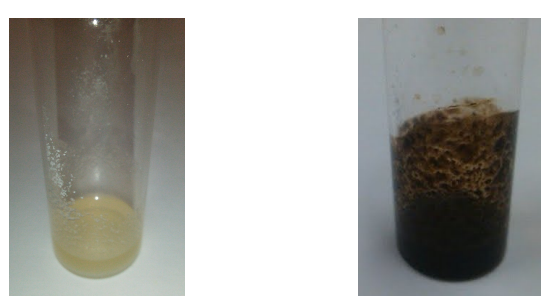

Figure 1: Demonstration of reactivity of KOtBu when mixed with (a) commercial 1,10-phenanthroline and (b) Purified 1,10-phenanthroline 
Given these observations, we decided to specifically examine the tert-butoxide-phenanthroline mixture by NMR spectroscopy. We were somewhat surprised to observe that mixing equimolar amounts of $\mathrm{KO} t \mathrm{Bu}$ with 1,10-phenanthroline leads to essentially complete destruction of the tert-butoxide moiety, the characteristic $9 \mathrm{H}$ singlet at $1.2 \mathrm{ppm}$ collapsing to $<1 \mathrm{H}$ according to the spectrum integral. The signals arising from the phenanthroline molecule are left untouched. The same result is obtained when $\mathrm{NaO} t \mathrm{Bu}$ and 1,10phenanthroline are mixed except the reaction takes $c a .5$ minutes rather than a few seconds (Figure 2).

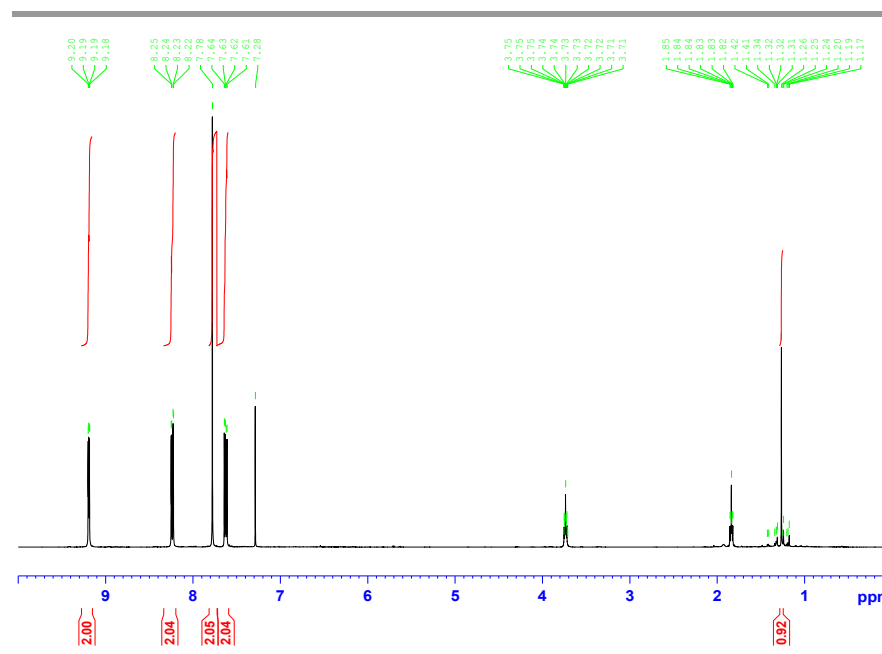

Figure 2: $1 \mathrm{H}$ NMR spectrum of an equimolar mixture of 1,10-phenanthroline and KOtBu demonstrating the collapse of the $9 \mathrm{H}$ singlet at $1.2 \mathrm{ppm}$.

Our model to explain this observation is one where the metal ion and alkoxide for the group 1 metals are in dynamic equilibrium between the essentially covalent and charge separated species. Additionally, when in the charge-separated form, the alkoxide can be thought of as being in equilibrium with a species that bears a loosely bound electron (Figure 3). At elevated temperatures this electron can be transferred to an aryl halide to initiate the reaction. This would tally with the work of Ashby, who demonstrated that the alkylation of alkoxides can proceed via single electron transfer from the alkoxide to the alkyl halide. ${ }^{10}$

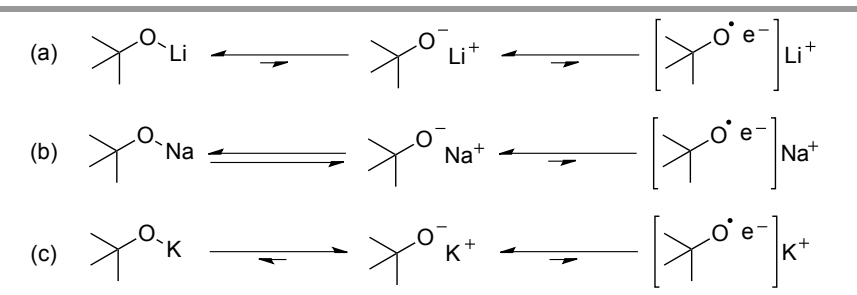

Figure 3: Increasing reducing power of group 1 alkoxides with increased cationic dissociation

The addition of 1,10-phenanthroline has an effect on the two equilibria (b) and (c) by removing the loosely bound electron from the complex and rendering the reaction irreversible. In the case of the lithium analogue (a), the initial equilibrium lies far to the left and the concentration of either of the dissociated species is too low for the reaction to be viable. The effect of adding phenanthroline to the system not only drives the equilibrium to the right, but by removing the loosely bound electron leaves a highly reactive alkoxy radical (Scheme 2).

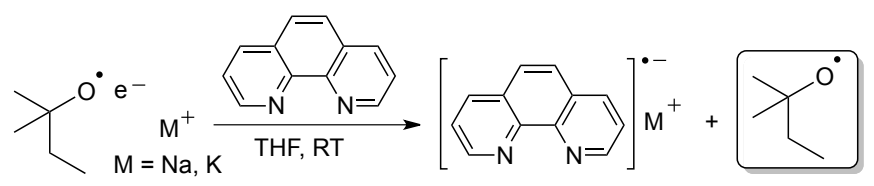

Scheme 2: Effect of phenanthroline on sodium and potassium pentoxide.

The alkoxy radical immediately collapses via a variety of wellknown decomposition pathways such as $\mathrm{H}$-atom abstraction and $\beta$ scission processes to yield highly reactive methyl radicals. ${ }^{11}$ The intractable mixtures of products and polymers which result from uncontrolled radical processes accounts for the immediate colour change on mixing the two reagents (Scheme 3).

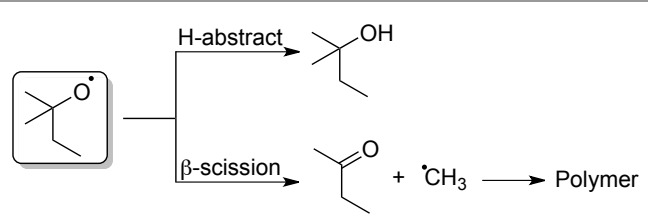

Scheme 3: Collapse of pentoxide radical to give butanone and methyl radicals

This we have confirmed by analysing the mixture of potassium pentoxide and 1,10-phenanthroline by mass spectrometry, which suggests that butanone is a major component of the reaction mixture. Aware of the difficulties in drawing conclusions from the mass spectra of low molecular weight compounds we therefore proceeded to confirm the presence of volatile, enolisable ketones in the reaction mixture, for which additional evidence was required. We therefore confirmed the collapse of the putative alkoxy radical by employing the established Janovsky Test for enolisable ketones. ${ }^{12}$ Addition of $m$-dinitrobenzene to a dilute THF solution of the alkoxidephenanthroline mixture resulted in an intense purple colour, indicating that the ketone was a significant component of the mixture (Figure 4).

(a)

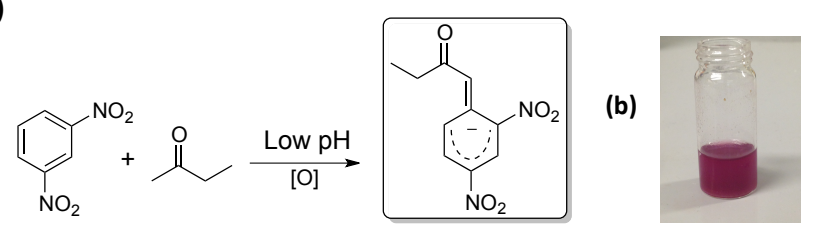

Figure 4: (a) Reaction of m-dinitrobenzene with butanone (Janovsky Test) and (b) (inset) Purple colour indicating the positive test obtained.

Given our evidence that increased charge separation between the metal cation and the alkoxy anion is responsible for the increasing reducing ability of the metal alkoxide (Figure 3), we expected that the addition of 15-crown-5 to sodium tert-butoxide would increase the concentration of charge separated species and result in a reaction broadly similar to that of the potassium analogue. Entry 11 in Table 1 demonstrates that this is clearly not the case. A consultation of the literature however reveals that although 15 -crown-5 assists in the solvation of sodium salts, simple stoichiometric mixtures maintain a contact association with the anion, particularly when the anion is highly coordinating. ${ }^{13,14}$ In this example it is unlikely therefore that the crown ether succeeds in generating sufficient levels of charge separated species to be synthetically useful (Figure 5). 


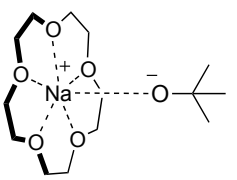

Figure 5: Contact association of alkoxide anion with sodium cations complexed by 15 -crown-5.

Presumably, once the alkoxy radical has collapsed the phenanthroline radical anion is then capable of transferring an electron to the aryl halide to initiate the radical coupling process. We conclude therefore that the phenanthroline derivatives serve as a temporary electron stock where they are sufficient electron acceptors to drive the equilibrium towards the alkoxy radical which then collapses, but do not hold the electron so tightly that it cannot be given up to an organic substrate in the reactions most recently reported. This is supported by the observation that 1,10phenanthroline does not appear to be consumed when mixed with potassium tert-butoxide whereas the alkoxide collapses rapidly. Others have noted that alternative additives, structurally unrelated to 1,10-phenanthroline (eg. macrocyclic pyridone pentamers developed by Zeng ${ }^{15}$ ) also promote the reaction in the same way. It is likely that these agents behave similarly, providing a haven for a loosely bound electron that can then be transferred to an organic substrate. Interestingly however, Murphy and Tuttle having examined similar processes by computational means concluded that electron transfer directly from the alkoxide to the aryl iodide is unlikely, in contrast to our results presented here. ${ }^{16}$ Our results have demonstrated that a 'ligand' or 'organocatalyst' is not necessarily an essential component of common so-called C-H activation reactions. The most important factor appears to be the degree of dissociation between the group 1 cation and the alkoxide, which can supply electrons to organic substrates when highly dissociated. Although many have attempted to describe these reactions in terms of catalytic cycles, our evidence suggests that a series of radical reactions is more accurate (where 1,10-phenanthroline is only required when the metal alkoxide is not sufficiently dissociated for the reaction to proceed) Scheme 6. This is in agreement with the conclusions of Studer and Curran who also describe such processes as "base promoted homolytic substitutions' ${ }^{17}$

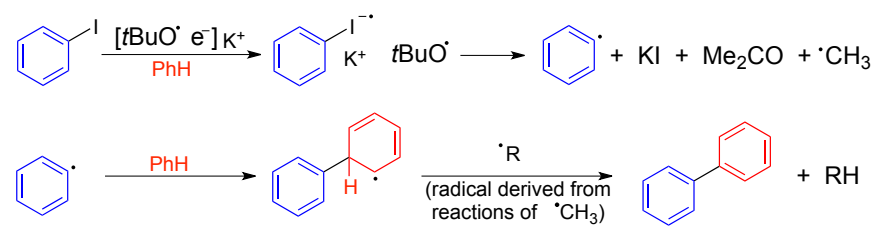

Scheme 6: Radical processes leading to biaryl formation.

\section{Conclusions}

In conclusion, we have described our experiments and observations on the TM free couplings of simple aryl systems. Our experiments show that the key to successful reactions (which are radical in nature) is the degree of dissociation of the metal counterion and the alkoxide. With the potassium (and presumably higher salts) the degree of dissociation is sufficient to allow the reaction to proceed without any additive. The addition of 1,10-phenanthroline facilitates the reaction only where the concentration of reactive species (on the right of the equilibria in Figure 3 ) is not sufficient for the reacti on to proceed. The results shed new light on these types of TM free coupling reaction. Although we now have a good understanding of the mechanism of these reactions and the role of 1,10 phenanthroline, the mechanism by which the diamines (eg DMEDA) appear to facilitate these processes is still unknown. Work is underway in our laboratory to understand this mechanism and to improve the efficiency of the reaction and will form the basis of future communications from our laboratory.

\section{Acknowledgements}

The authors would like to thank UCL for support via the doctoral training programme in Organic Chemistry: Drug Discovery.

\section{Notes and references}

a Department of Chemistry, University College London, 20 Gordon Street, London, WC1H 0AJ, UK. Email: j.wilden@ucl.ac.uk Electronic Supplementary Information (ESI) available: Full characterisation and analytical data is provided. See DOI: $10.1039 / \mathrm{c} 000000 \mathrm{x} /$

1 C-L. Sun, H. Li, D-G. Yu, M. Yu, X. Zhou, X-Y. Lu, K. Huang, S-F. Zheng, B. J. Li, Z-J. Shi, Nature Chem. 2010, 2, 1044-1049.

2 E. Shirakawa, K-I. Itoh, T. Higashino, T. Hayashi, J. Am. Chem. Soc. 2010, 132, 15537-15539.

3 W. Liu, H. Cao, H. Zhang, H. Zhang, K. H. Chung, C. He, H. Wang, F. Y. Kwong, A. Lei, J. Am. Chem. Soc. 2010, 132, 16737-16740.

4 M. Rueping, M. Leiendecker, A. Das, T. Poisson, L. Bui, Chem. Commun. 2011, 47, 10629-10631.

5 G. Jin, X. Zhang, S. Cao, Org. Lett. 2013, 15, 3114-3117.

6 R. K. Arvela, N. E. Leadbeater, M. S. Sangi, V. A. Williams, P. Granados, R. D. Singer, J. Org. Chem. 2005, 70, 161-168.

7 S. Yanagisawa, K. Ueda, T. Taniguchi, K. Itami, Org. Lett. 2008, 10, 4673-4676.

8 V. J. Gray, B. Slater, J. D. Wilden, Chem. Eur. J. 2012, 18, 1558215585.

9 E. Schmidt, A. Hourdakis, A. I. Popov, Inorg. Chim. Acta. 1981, 52, 91-95.

10 E. C. Ashby, Acc. Chem. Res. 1988, 21, 414-421.

11 P. Gray, A. Williams, Chem. Rev. 1959, 59, 239-328.

12 R. J. Pollitt, B. C. Saunders, J. Chem. Soc. 1965, 4615-4628.

13 G. Lindsten, O. Wennerström, B. Thulin, Acta. Chem. Scand. B, 1986, 40, 545-554.

14 N. S. Poonia, M. R. Truter, J. Chem. Soc. Dalton. Trans. 1973, 20622065.

15 H. Zhou, J. Shen, J. Guo, R. Ye, H. Zeng, Chem. Commun. 2013, 49, 2323-2325.

16 S. Zhou, G. M. Anderson, B. Mondal, E. Doni, V. Ironmonger, M. Kranz, T. Tuttle, J. A. Murphy, Chem. Sci. 2013, DOI: $10.1039 / \mathrm{c} 3 \mathrm{sc} 52315 \mathrm{~b}$

17 A. Studer, D. P. Curran, Angew. Chem. Int. Ed. 2011, 50, 5018-5022. 